\title{
Conhecimento e atitudes de trabalhadores de um serviço público de emergência sobre adoção de precauções padrão
}

\author{
Knowledge and attitudes of workers from a public emergency service about the \\ adoption of standard precautions
}

Conocimientos y actitudes de trabajadores de un servicio de emergencia pública en relación con la adopción de precauciones estándar

\author{
Maria Henriqueta Rocha Siqueira Paiva', Adriana Cristina de Oliveira" \\ ' Centro Universitário Metodista Izabela Hendrix, Curso de Enfermagem. \\ Serviço de Atendimento Móvel de Urgência. Belo Horizonte, Minas Gerais, Brasil. \\ "Universidade Federal de Minas Gerais, Escola de Enfermagem. Belo Horizonte, Minas Gerais, Brasil.
}

Submissão: 22/12/2009 Revisão: 20/3/2011 Aprovação: 15/5/2011

\section{RESUMO}

Estudo transversal realizado em um serviço de atendimento pré-hospitalar de Minas Gerais, com o qual se objetivou avaliar a adoção das medidas de precaução, por meio de conhecimento e atitudes da equipe mutiprofissional. Utilizou-se análise univariada e multivariada dos dados. Verificou-se que enfermeiros e condutores apresentaram o maior e o menor nível de conhecimento em relação às precauções padrão, respectivamente. A não adoção das medidas de precaução foi 3,76 (IC 95\%: 1,48 - 9,53) vezes maior entre profissionais com idade superior a 31 anos e 6,7 (IC 95\%: 1,81 - 24,75) vezes maior entre trabalhadores de unidade de suporte básico. Diante destes resultados, torna-se imprescindível implantar um programa de educação continuada para melhorar o conhecimento do profissional em relação ao controle de infecção e recomendações da biossegurança.

Descritores: Serviços médicos de emergência; Conhecimento; Atitude; Equipe de assistência ao paciente.

\begin{abstract}
The aims of the study were to evaluate the adoption of the precaution measures, assessing knowledge and attitude of multiprofessional team. This is a transversal study, accomplished in an Emergency Medical Service of Minas Gerais. Univariate and multivariate analysis were used. The results showed that nurses and drivers had the highest and lowest level of knowledge regarding the standard precautions, respectively. The possibility of non-adoption of the precautionary measures was $3.76(95 \%$ $\mathrm{Cl}$ : 1.48 to 9.53) times higher among professionals aged over 31 years and 6.7 (95\% Cl: 1.81 to 24,75) times greater among workers in crowded unit of basic support. The conclusion is that is essential to implement strategies in order to improve the professional's knowledge related to infection control and safety recommendations.
\end{abstract}

Descriptors: Emergency medical services; Knowledge; Attitude; Patient care team.

\section{RESUMEN}

Estudio transversal, realizado en un servicio de atención pre-hospitalaria de Minas Gerais, cuyo objetivo fue evaluar la adopción de medidas de precaución, por medio del conocimiento y de las actitudes del equipo multiprofesional. Se utilizó el análisis univariada y multivariada de los datos. Se encontró que los enfermeros y los conductores han tenido el nivel más alto y el más bajo e conocimiento en relación a las precauciones estándar, respectivamente. La no adopción de medidas de precaución fue de 3,76 (IC 95\%: 1,48 a 9,53) veces mayor para los profesionales de 31 años y 6,7 (IC 95\%: 1,81 a 24,75) veces superior entre los trabajadores en la unidad de soporte básico. Teniendo en cuenta estos resultados, es esencial establecer un programa de educación continuada para mejorar los conocimientos profesionales en relación con el control de infecciones y las recomendaciones de bioseguridad.

Palabras clave: Servicio médico de emergencia; Conocimiento; Actitud; Servicios de atención al paciente. 


\section{INTRODUÇÃO}

O serviço de urgência e emergência público também chamado de atendimento pré-hospitalar (APh) oferece cuidados de saúde imediatos às vítimas de mal súbito ou traumas até a chegada a uma instituição especializada ${ }^{(1)}$. Nos últimos anos, verificou-se um aumento pela procura de serviços de emergência em todo o mundo devido ao grande número de acidentes, violência e doenças agudas ${ }^{(1-2)}$. Este fato estimulou a rápida expansão dos serviços de emergência, e, por conseguinte, a necessidade de que órgãos como o Centers for Disease Control And Prevention (CDC) voltasse sua atenção para aspectos da segurança e saúde dos trabalhadores nessa modalidade de trabalho(3).

Na maioria das vezes, o serviço de urgência e emergência, por sua própria dinâmica, favorece a maior exposição dos profissionais ao contato com fluidos corporais, passíveis de transmissão de doenças, especialmente infecções virais como a Hepatite $\mathrm{B}$ e C, e a síndrome de imunodeficiência adquirida ${ }^{(3.4)}$.

O ritmo acelerado de trabalho dos profissionais, bem como a não utilização das medidas de precaução, tais como a utilização de equipamento de proteção individual (EPI), lavagem das mãos, vacinação contra hepatite $B$, e descarte adequado de material pérfuro-cortante, são importantes causas que podem intensificar os riscos de acidentes com trabalhadores da saúde ${ }^{(5.6)}$.

De acordo com resultados de alguns trabalhos ${ }^{(6-8)}$, a não adoção das medidas de precaução está relacionada ao conhecimento e atitude dos profissionais. Entre as causas listadas destacam-se a falta de conhecimento na área da biossegurança e controle de infecções; a preparação psicológica insuficiente para lidar com situações relacionadas à assistência fora do ambiente hospitalar especializado; necessidade de realização de intervenções rápidas e eficazes em curtos períodos de tempo; desconhecimento da situação de saúde do paciente; e pela crença de que faz parte do trabalho em emergência conviver com situações de risco.

Diante de escassez de estudos sobre a exposição do profissional do atendimento de emergência pré-hospitalar aos riscos cotidianos de sua atividade, esse estudo objetivou analisar o conhecimento e a atitude de tais profissionais em relação à adoção às medidas de precaução, aos riscos ocupacionais e à atenção às recomendações básicas de controle de infecção.

\section{MATERIAL E MÉTODOS}

Trata-se de um estudo de delineamento transversal, realizado com equipe multiprofissional do serviço de emergência pré-hospitalar da região metropolitana de Belo Horizonte. Os dados foram colhidos entre junho e novembro de 2006, no município de Contagem-MG, Brasil.

Foi aplicado um questionário estruturado que incluiu os seguintes itens: dados demográficos (profissão, sexo, idade, o tempo decorrido desde graduação profissional, experiência profissional no serviço público de emergência e unidade de lotação) e questões relativas ao conhecimento, atitude, controle de infecção e precauções padrão.

O conhecimento foi definido como informação, entendimento formal ou noção sobre controle de infecção e recomendações relacionadas à biossegurança ${ }^{(9)}$. As informações sobre o conhecimento adequado foram interpretadas de acordo com os princípios que orientam a prática de uma assistência de qualidade, visando à redução dos riscos profissionais. Além disso, utilizaram-se as referências relativas à segurança do trabalho e medidas de precauções padrão recomendadas pelo Centers for Disease Control and Prevention - $\mathrm{CDC}^{(3)}$. Considerou-se conhecimento inadequado toda informação sobre assuntos específicos relacionados a precauções padrão e controle de infecção que não se encontravam em concordância com a literatura científica ${ }^{(3)}$.

Em relação à adoção de medidas de precaução e controle de infecção, foi analisado o conhecimento sobre: veículo de transmissão de agentes infecciosos; indicação de uso de luvas descartáveis; higienização das mãos antes e após o contato com o paciente; risco para infecção tendo como porta de entrada do agente infeccioso a mucosa ocular; risco para infecção cruzada durante realização de acesso venoso; doenças que podem ser contraídas pela natureza do trabalho realizado no atendimento pré-hospitalar; precauções padrão específicas para o atendimento de emergência; acondicionamento dos resíduos produzidos durante o atendimento; conduta adequada do profissional pós-acidente de trabalho com exposição a material biológico; e esquema vacinal completo para hepatite $\mathrm{B}$.

A atitude foi definida como a conduta do profissional em relação aos princípios do controle de infecção e recomendações sobre biossegurança durante $\mathrm{o}$ atendimento emergencial ${ }^{(8)}$. A resposta à atitude adequada foi verificada pelo comportamento esperado durante o serviço realizado em consonância com os princípios orientadores da prática de cuidados de saúde com qualidade e redução de riscos profissionais, de acordo com as referências sobre segurança no trabalho e medidas de precauções padrão recomendadas pelo $\operatorname{CDC}^{(1,3,4,8)}$. Para analisar a atitude em relação às medidas de precaução, foram selecionados os seguintes tópicos: medidas para reduzir a transmissão de agentes infecciosos; indicação para a utilização de máscaras e óculos de proteção; descarte apropriado do material pérfuro-cortante e uso adequado de equipamento de proteção individual durante o serviço de emergência.

Foi utilizado instrumento de coleta de dados validado por peritos nas áreas de controle de infecção hospitalar, epidemiologia e de saúde do trabalhador. Definiu-se como indicador geral de "bom conhecimento", e "boa atitude", índice de 75\% de respostas adequadas relacionadas ao conhecimento e as atitude ${ }^{(9)}$.

Os resultados foram analisados de acordo com a categoria profissional (médicos, enfermeiros, técnicos / auxiliares de enfermagem, e condutores), idade, sexo, tempo de formação profissional, tempo de atuação na instituição, e unidade de lotação.

Foram utilizados testes qui-quadrado para comparar as variáveis categóricas. Para avaliar possíveis associações entre a adoção de medidas de precaução e as variáveis categóricas foi realizada análise univariada, usando a regressão logística. Para todos os testes, foi considerado estatisticamente significativo o valor de $p$ inferior a 0,05 . Os dados foram analisados pelo software SPSS (pacote estatístico para as ciências sociais), versão 13.5.

O estudo proposto foi apresentado à Secretaria de Saúde do município de Contagem sendo autorizada a aplicação dos questionários aos profissionais do APh. Os profissionais da 
equipe foram abordados individualmente, sendo-lhes esclarecidos os objetivos e a finalidade do estudo. Os respondentes aceitaram participar do estudo através da assinatura do termo de consentimento livre e esclarecido, sendo, assim, respeitados os preceitos da Resolução 196/96 do Conselho Nacional de Saúde, que regulamenta pesquisa com seres humanos.

\section{RESULTADOS}

Entre os participantes do estudo $(\mathrm{N}=116), 15,5 \%$ eram médicos, 7,8\% enfermeiros, 55,2\% técnicos / auxiliares de enfermagem e 21,5 condutores. A maioria dos profissionais era do sexo masculino (56,0\%), graduados antes do ano 2000 $(57,1 \%)$, com tempo de atuação na instituição superior a um ano $(59,5 \%)$ e lotados em unidades de emergência $(70,7 \%)$ cujos procedimentos são caracterizados como de menor complexidade tais como acesso venoso periférico; aspiração de conteúdo oral; contenção de hemorragias por outras lesões; dentre outras. Em relação à categorização dos trabalhadores por faixa etária não foi observada diferenças significativas, sendo a idade de 31 anos a mediana desta população.

Pode-se observar na Tabela 1 que os médicos obtiveram $83,3 \%$ de respostas adequadas em relação aos itens analisados. No entanto, para os itens relacionados às precauções padrão, indicação de higienização das mãos ao cuidar de qualquer paciente e acondicionamento adequado do resíduo produzido durante o atendimento, o índice de conhecimento foi inferior a $75 \%$.

Os enfermeiros alcançaram $83,8 \%$ em média de respostas adequadas em relação aos itens avaliados; porém, para a questão relacionada ao conhecimento sobre precauções padrão

Tabela 1: Distribuição das respostas relacionadas ao conhecimento das medidas de precaução padrão segundo a categoria profissional. Contagem-MG, Brasil, 2006.

\begin{tabular}{|c|c|c|c|c|c|}
\hline Conhecimento & $\begin{array}{c}\text { Médico } \\
\begin{array}{c}\text { N=18 } \\
\%\end{array}\end{array}$ & $\begin{array}{c}\text { Enfermeiro } \\
\begin{array}{c}N=9 \\
\%\end{array}\end{array}$ & $\begin{array}{c}\text { Téc/aux de enf. } \\
\begin{array}{c}N=64 \\
\%\end{array}\end{array}$ & $\begin{array}{c}\text { Condutor } \\
\begin{array}{c}N=25 \\
\%\end{array}\end{array}$ & p-valor \\
\hline Veículo mais importante de transmissão de microrganismos & $\begin{array}{c}17 \\
(94,4)\end{array}$ & $\begin{array}{c}9 \\
(100,0)\end{array}$ & $\begin{array}{c}58 \\
(90,6)\end{array}$ & $\begin{array}{c}14 \\
(56,0)\end{array}$ & $0,001 *$ \\
\hline $\begin{array}{l}\text { Indicação de uso de luvas descartáveis no } \\
\text { atendimento pré-hospitalar }\end{array}$ & $\begin{array}{c}17 \\
(94,4)\end{array}$ & $\begin{array}{c}9 \\
(100,0)\end{array}$ & $\begin{array}{c}50 \\
(78,1)\end{array}$ & $\begin{array}{c}13 \\
(52,0)\end{array}$ & 0,002 \\
\hline Importância da lavagem das mãos & $\begin{array}{c}16 \\
(88,9)\end{array}$ & $\begin{array}{c}7 \\
(77,8)\end{array}$ & $\begin{array}{c}54 \\
(84,4)\end{array}$ & $\begin{array}{c}13 \\
(52,0)\end{array}$ & 0,006 \\
\hline $\begin{array}{l}\text { Indicação de higienização das mãos ao cuidar } \\
\text { de qualquer paciente }\end{array}$ & $\begin{array}{c}13 \\
(72,2)\end{array}$ & $\begin{array}{c}8 \\
(88,9)\end{array}$ & $\begin{array}{c}57 \\
(89,1)\end{array}$ & $\begin{array}{c}17 \\
(68,0)\end{array}$ & $0,064^{*}$ \\
\hline $\begin{array}{l}\text { Risco de infecção por contato de sangue } \\
\text { com a mucosa ocular }\end{array}$ & $\begin{array}{c}16 \\
(88,9)\end{array}$ & $\begin{array}{c}9 \\
(100,0)\end{array}$ & $\begin{array}{c}28 \\
(48,4)\end{array}$ & $\begin{array}{c}8 \\
(32,0)\end{array}$ & $<0,001$ \\
\hline $\begin{array}{l}\text { Risco de transmissão cruzada de agentes infecciosos } \\
\text { ao realizar punção de acesso venoso periférico }\end{array}$ & $\begin{array}{c}14 \\
(77,8)\end{array}$ & $\begin{array}{c}7 \\
(77,8)\end{array}$ & $\begin{array}{c}41 \\
(64,1)\end{array}$ & $\begin{array}{c}16 \\
(64,0)\end{array}$ & 0,621 \\
\hline $\begin{array}{l}\text { Possíveis infecções que podem ser contraídas } \\
\text { pelo contato com fluídos corporais e sangue }\end{array}$ & $\begin{array}{c}16 \\
(88,9)\end{array}$ & $\begin{array}{c}7 \\
(77,8)\end{array}$ & $\begin{array}{c}44 \\
(68,8)\end{array}$ & $\begin{array}{c}16 \\
(64,0)\end{array}$ & 0,287 \\
\hline Precauções padrão para o atendimento pré-hospitalar & $\begin{array}{c}13 \\
(72,2)\end{array}$ & $\begin{array}{c}5 \\
(55,6)\end{array}$ & $\begin{array}{c}33 \\
(51,6)\end{array}$ & $\begin{array}{c}21 \\
(84,0)\end{array}$ & 0,029 \\
\hline $\begin{array}{l}\text { Acondicionamento adequado dos resíduos } \\
\text { produzidos no atendimento pré-hospitalar }\end{array}$ & $\begin{array}{c}12 \\
(66,7)\end{array}$ & $\begin{array}{c}7 \\
(77,8)\end{array}$ & $\begin{array}{c}50 \\
(78,1)\end{array}$ & $\begin{array}{c}23 \\
(92,0)\end{array}$ & 0,233 \\
\hline $\begin{array}{l}\text { Medidas após acidente de trabalho envolvendo } \\
\text { materiais pérfuro-cortantes ou fluídos corporais }\end{array}$ & $\begin{array}{c}16 \\
(88,9)\end{array}$ & $\begin{array}{c}7 \\
(77,8)\end{array}$ & $\begin{array}{c}57 \\
(89,1)\end{array}$ & $\begin{array}{c}20 \\
(80,0)\end{array}$ & $0,503 *$ \\
\hline Vacinação para Hepatite B & $\begin{array}{c}18 \\
(100,0)\end{array}$ & $\begin{array}{c}8 \\
(88,9)\end{array}$ & $\begin{array}{c}55 \\
(85,9)\end{array}$ & $\begin{array}{c}19 \\
(76,0)\end{array}$ & $0,129 *$ \\
\hline Média & $\begin{array}{c}15 \\
(83,3)\end{array}$ & $\begin{array}{c}8 \\
(83,8)\end{array}$ & $\begin{array}{c}48 \\
(75,0)\end{array}$ & $\begin{array}{c}16 \\
(64,0)\end{array}$ & \\
\hline
\end{tabular}

Fonte: Dados da pesquisa.

* Teste exato de Fischer 
apropriadas ao atendimento emergencial, o nível de respostas adequadas foi inferior a $75 \%$. Os técnicos e auxiliares de enfermagem também não atingiram $75 \%$ de respostas adequadas nas questões relacionadas à infecção cruzada, precauções padrão, doenças transmissíveis pelo sangue, bem como o risco de infecção por contato entre o sangue e a mucosa ocular.

Os condutores apresentaram baixo nível de conhecimento, quando comparado aos outros profissionais, pontuando em média $64,0 \%$ (respostas adequadas), revelaram falta de conhecimento sobre quase todos os itens avaliados, exceto para a indicação de higienização das mãos, precauções padrão apropriadas ao atendimento pré-hospitalar, acondicionamento adequado dos resíduos produzidos durante o atendimento, cuidados pós-acidentes com material pérfuro-cortante e vacinação para hepatite $B$.

Observou-se diferença estatisticamente significante entre as categorias profissionais quanto ao veículo de transmissão de agentes infecciosos; à indicação para a utilização de luvas descartáveis no serviço de emergência; importância da higienização das mãos; risco de infecção por contacto entre o sangue e mucosa ocular; e precauções padrão apropriadas ao atendimento emergencial $(p<0,01)$.

A Tabela 2 mostra a proporção de respostas adequadas relacionados à atitude referentes às precauções padrão. Verificou-se que, apesar de todas as categorias terem alcançado um índice de respostas adequadas superior a $75 \%$, foi observado que para o item indicação do uso de máscara facial e óculos de proteção em pacientes que eliminam sangue por via aérea apresentou associação estatisticamente significante, sendo que técnicos e auxiliares de enfermagem apresentaram médias de respostas adequadas inferior aos médicos e enfermeiros.

Foi encontrada, também, associação estatisticamente significante em relação ao descarte adequado de material pérfuro-cortante, sendo que o percentual de respostas adequadas de todos os profissionais de saúde foi maior que o verificado entre condutores.

Observou-se, também, quanto à atitude do profissional em relação ao uso adequado do equipamento de proteção individual (EPI) durante o atendimento pré-hospitalar, que apenas os condutores atingiram um percentual superior a $75 \%$ de respostas adequadas.

$\mathrm{Na}$ analise da regressão logística multivariada, considerando-se as variáveis demográficas, observou-se que profissionais com mais de 31 anos ( $p=0,005)$ e lotados em unidades de suporte básico $(p=0,004)$ estiveram estatisticamente associados com a não adoção às medidas de precaução. A probabilidade estimada de não adotar medidas de precaução foi 3,76 (IC 95\%: 1,48-9,53) vezes maior entre profissionais com idade superior a 31 anos, em comparação aos respondentes com idade inferior; e 6,7 (IC 95\%: 1,81-24,75) vezes maior nos trabalhadores lotados em unidade de suporte básico se comparados aos trabalhadores da unidade de suporte avançado.

\section{DISCUSSÃO}

A distribuição de profissionais da equipe de emergência observada neste estudo se encontra em conformidade com aquela preconizada pela Portaria $n^{\circ} 2.048 / \mathrm{MS}$, que estabelece os princípios, diretrizes, normas e critérios de funcionamento dos serviços de Urgência e Emergência no Brasil(5).

Com relação à distribuição por sexo, pode-se inferir que a maior concentração de profissionais do sexo masculino neste serviço se deve à preferência de tal gênero em virtude da especificidade do atendimento, exemplificado pela diversidade de atividades prestadas durante a assistência à saúde, pela clientela (indivíduos obesos, gestantes, dentre outros) e pela sobrecarga física necessária ao transporte de equipamentos durante o atendimento. Este dado vem corroborar os

\section{Tabela 2: Distribuição das respostas analisadas sobre à atitude em relação às medidas de precaução padrão segundo a categoria profissional. Contagem-MG, Brasil, 2006.}

\begin{tabular}{|c|c|c|c|c|c|}
\hline Atitude & $\begin{array}{c}\text { Médico } \\
\begin{array}{c}N=18 \\
\%\end{array}\end{array}$ & $\begin{array}{c}\text { Enfermeiro } \\
\qquad \begin{array}{c}N=9 \\
\%\end{array}\end{array}$ & $\begin{array}{c}\text { Téc. / Aux de enf. } \\
\qquad \begin{array}{c}N=64 \\
\%\end{array}\end{array}$ & $\begin{array}{c}\text { Condutor } \\
N=25 \\
\%\end{array}$ & $p$-valor \\
\hline $\begin{array}{l}\text { Medidas para redução de transmissão } \\
\text { de agentes infecciosos }\end{array}$ & $\begin{array}{c}14 \\
(77,8)\end{array}$ & $\begin{array}{c}7 \\
(77,8)\end{array}$ & $\begin{array}{c}54 \\
(84,4)\end{array}$ & $\begin{array}{c}19 \\
(76,0)\end{array}$ & $0,703 *$ \\
\hline $\begin{array}{l}\text { Indicação do uso de máscara facial e } \\
\text { óculos de proteção em pacientes que } \\
\text { eliminam sangue por via aérea }\end{array}$ & $\begin{array}{c}17 \\
(94,4)\end{array}$ & $\begin{array}{c}9 \\
(100,0)\end{array}$ & $\begin{array}{c}52 \\
(81,3)\end{array}$ & $\begin{array}{c}25 \\
(100,0)\end{array}$ & $0,044^{*}$ \\
\hline Descarte adequado de material pérfuro-cortante & $\begin{array}{c}17 \\
(94,4)\end{array}$ & $\begin{array}{c}9 \\
(100,0)\end{array}$ & $\begin{array}{c}63 \\
(98,4)\end{array}$ & $\begin{array}{c}10 \\
(40,0)\end{array}$ & $<0,001^{*}$ \\
\hline $\begin{array}{l}\text { EPI adequado para uso durante } \\
\text { o atendimento pré-hospitalar }\end{array}$ & $\begin{array}{c}10 \\
(55,6)\end{array}$ & $\begin{array}{c}4 \\
(44,4)\end{array}$ & $\begin{array}{c}41 \\
(64,1)\end{array}$ & $\begin{array}{c}19 \\
(76,0)\end{array}$ & 0,308 \\
\hline Média & $\begin{array}{c}14 \\
(78,0)\end{array}$ & $\begin{array}{c}7 \\
(72,5)\end{array}$ & $\begin{array}{c}53 \\
(82,0)\end{array}$ & $\begin{array}{c}18 \\
(72,0)\end{array}$ & \\
\hline
\end{tabular}

Fonte: Dados da pesquisa.

*Teste exato de Fischer 
resultados obtidos em estudos brasileiros com equipe multiprofissional de atendimento pré-hospitalar ${ }^{(9,10)}$.

Quanto à faixa etária, observou-se uma distribuição equitativa em conformidade com a mediana da amostra estudada (31 anos), a idade dos profissionais variou de 23 a 59 anos. Este dado também pode ser observado na maioria dos estudos realizados com equipes de atendimento emergencial. Pelas características do atendimento emergencial, a predominância de profissionais jovens, provavelmente, se dê em função de sua melhor disponibilidade pessoal, física e profissional.

No que diz respeito ao nível de conhecimento dos profissionais, constatou-se que apenas condutores obtiveram um índice superior a $75 \%$ de respostas adequadas na questão relacionada às medidas de precauções inerentes ao serviço de atendimento de emergência. Também verificou-se associação estatisticamente significativa em questões relacionadas ao conhecimento especifico de patógenos veiculados pelo sangue, indicação de uso de luvas descartáveis no atendimento emergencial, importância da lavagem das mãos, indicação de higienização das mãos ao cuidar de qualquer paciente, sendo a média de respostas adequadas entre condutores menor que a média dos demais profissionais. Pode-se inferir que, durante a formação profissional, nem sempre são oferecidos conteúdos relacionados ao atendimento de emergência, e a carência da educação continuada em serviço dificultaria o acesso dos profissionais às orientações e consequente implementação, destas, à prática ${ }^{(8,11)}$.

$\mathrm{Na}$ análise do conhecimento por categoria, verificou-se que os profissionais apresentaram níveis inferiores a $75 \%$, de respostas adequadas, em itens distintos (Tab. 1). A diferença no nível de conhecimento entre os profissionais do atendimento emergencial pode ser parcialmente explicada pelas diferentes abordagens na formação acadêmica destes. Para o médico é necessário um conhecimento aprofundado sobre etiologia, formas de transmissão, disseminação e prevenção de doenças. Para o enfermeiro, a ênfase concentra-se nas manifestações e como cuidar do paciente em cada fase do processo saúde/doença. Já, para o condutor, exige-se a habilidade para trafegar com rapidez e segurança. Entretanto, neste tipo de atendimento, esse também participa, realizando procedimentos de menor complexidade, como massagem cardíaca externa, contenção de hemorragias e imobilização de fraturas, expondo-se, dessa forma, aos mesmos riscos ocupacionais que os demais profissionais.

Neste estudo, observou-se que o conhecimento sobre as precauções padrão entre médicos, técnicos/auxiliares de enfermagem e enfermeiros atingiu índices inferiores a $75 \%$. Evidenciou-se, assim, pelas respostas obtidas, que estes profissionais desconhecem as precauções padrão para o atendimento de emergência, ou seja: uso de equipamento de proteção individual adequado, higienização das mãos, imunização para hepatite $B$, descarte do material pérfuro-cortante em recipiente de paredes rígidas, e acondicionamento do lixo produzido durante $o$ atendimento.

Esses resultados corroboram estudo em que se observou que, apesar dos profissionais relatarem conhecimento e adoção às precauções padrão, a adoção efetiva destas não foi constatada na prática ${ }^{(11)}$. Em outro estudo, $45,9 \%$ da amostra analisada possuía conhecimento adequado sobre precauções padrão; 87,8\% concordaram com a possibilidade de infecções cruzadas; $73,0 \%$ relataram a importância do protetor ocular para evitar contaminação pela mucosa ocular; e apenas $13,5 \%$ sabiam quais infecções podiam ser transmitidas pelo contato com sangue ${ }^{(11)}$.

Para a avaliação do acondicionamento final dos resíduos produzidos pelo atendimento de emergência, averiguou-se, pelas respostas obtidas, que somente os médicos desconheciam que os resíduos resultantes da atenção à saúde de indivíduos devem ser acondicionados em saco branco leitoso. Este fator também pode estar associado à carência de um protocolo que estabeleça como o profissional deva proceder para acondicionar os resíduos corretamente ${ }^{(12)}$.

Em relação à indicação do uso de luvas descartáveis durante $o$ atendimento emergencial, verificou-se que profissionais condutores apresentaram um percentual inferior a $75 \%$ de respostas adequadas sobre $\mathrm{o}$ assunto. $\mathrm{O}$ uso de luvas descartáveis, como parte das precauções padrão durante o atendimento, é indicado para proteger o trabalhador do contato com sangue e fluídos corporais ${ }^{(4)}$.

Tal fato se agrava quando se percebe a ausência de formação do condutor na área da saúde e de treinamentos em técnicas básicas. Apesar de trabalhar juntamente com a equipe de saúde durante o atendimento emergencial, nem sempre é possível observar se o conhecimento destes profissionais é repassado ao condutor ou que este o esteja adotando em sua prática. Com isso, pode-se inferir que, muitas vezes, a utilização das luvas pelos condutores ocorre apenas com o propósito de se proteger, sem que o mesmo tenha conhecimento da possibilidade deste material ser capaz de transmitir contaminação / colonização entre pacientes, profissionais, equipamentos e ambiente.

Segundo o Centers for Disease Control and Prevention, qualquer trabalhador em serviços de emergência, e cujo trabalho exige contato com sangue ou outros fluidos corporais deve receber informações sobre biossegurança durante o horário de trabalho e participar de cursos anuais $\mathrm{s}^{(3,13)}$.

Além disso, programas que visem educação em serviço devem ser incentivados, desenvolvidos e implementados com a finalidade de reduzir as diferenças individuais de conhecimento entre a equipe de saúde ${ }^{(3)}$.

$\mathrm{Na}$ análise de atitude, apenas o condutor atingiu $75 \%$ de respostas adequadas para a indicação de EPI adequado a todo atendimento emergencial. Este resultado chamou atenção, por este trabalhador não ser considerado profissional de saúde; e, por ser esperado que os demais integrantes da equipe tenham recebido, durante a fase de formação profissional e treinamentos, orientações sobre o tema. Pode-se inferir que o receio pelo desconhecido faça com que este profissional reconheça a necessidade de utilizar o EPI mesmo sem conhecimento de sua indicação precisa.

O uso do EPI é essencial para a proteção do profissional do serviço de atendimento à emergências principalmente fora do ambiente hospitalar. Embora o uso de proteção individual não impeça que o trabalhador sofra o acidente, favorece de alguma forma que a exposição ao risco seja menor ${ }^{(14-15)}$. Alguns autores relatam que a realidade da adoção das medidas de precauções 
está longe de ser aquilo que é recomendada(16-17), porém em estudo realizado nos Estados Unidos com paramédicos verificou-se que no Estado da Califórnia, onde existe legislação específica sobre manipulação de pérfuro-cortantes e que também durante a realização do estudo foram disponibilizados materiais de proteção individual em maior quantidade, os profissionais apresentaram uma maior adoção às medidas de precaução ${ }^{(15)}$.

Como o conhecimento e atitude em relação a noções básicas de controle de infecção e a precauções padrão foram utilizadas, neste estudo, para analisar a adoção às precauções padrão entre a equipe multiprofissional do atendimento emergencial, a categoria que demonstrou maior conhecimento teve também maior chance de adotar os princípios da biossegurança, e vice-versa. Ou seja, como o enfermeiro e o médico apresentaram os maiores índices de respostas corretas em relação ao assunto analisado, estes tiveram mais chance em relação aos outros de adotar as precauções padrão.

A técnica da regressão logística multivariada revelou uma associação estatisticamente significativa entre a não adoção às precauções padrão e profissionais com mais de 31 anos de idade, lotados em unidades que realizam procedimentos de menor complexidade, $p<0,05$. A diferença de formação acadêmica entre a equipe de profissionais que compõem uma unidade de atendimento avançado em relação a uma unidade de atendimento básico poderia justificar os resultados encontrados nesta distribuição, uma vez que a primeira é composta por dois profissionais de nível universitário e a segunda por dois profissionais de nível médio. Com relação à idade, verificou-se que a faixa etária dos profissionais que trabalham em atendimento emergencial corrobora os resultados desta pesquisa. Alguns autores registraram a ocorrência deste evento relacionada à sobrecarga de trabalho que, além de deixar o profissional mais estressado, exige maior rapidez na execução das tarefas e, muitas vezes, induz a um fazer repetitivo sem adequado planejamento ${ }^{(5,10,15)}$.

\section{CONCLUSÃO}

Ao ser utilizado como indicador geral de "bom conhecimento" e "boa atitude" um índice de 75\% de respostas adequadas relacionadas ao conhecimento e à atitude, verificou-se que médicos, enfermeiros, técnicos / auxiliares de enfermagem apresentaram média de respostas adequadas ao conhecimento em relação às precauções padrão. Porém, quando foram analisadas questões sobre atitude, observou-se que enfermeiros e condutores obtiveram média inferior a $75 \%$ de respostas adequadas, enquanto para médicos e técnicos / auxiliares de enfermagem a média obtida foi superior a $75 \%$.

Para o aspecto do conhecimento, o condutor foi o profissional que apresentou os menores índices de respostas adequadas em quase todos os itens avaliados. Vale ressaltar que, dentro da equipe, além de conduzir o veículo de transporte, este profissional exerce também a função de socorrista, embora não seja considerado um profissional de saúde. Ao realizar procedimentos de suporte básico de vida, junto à equipe de saúde, descartar material pérfuro-cortante utilizado durante o atendimento e higienizar o veículo, esses estariam tão sujeitos ao risco de acidente ocupacional com exposição a material biológico quanto os demais profissionais que compõem a equipe multiprofissional.

Pode-se constatar, também, que os profissionais desta equipe apresentaram déficits diferentes em relação ao conhecimento, sendo importante abordar esta equipe por dificuldades comuns relacionadas ao conhecimento; e, cada categoria, separadamente, por dificuldades especificas.

A conclusão da necessidade de um programa de educação continuada parece óbvia, pois, em um serviço que pretenda manter padrões adequados de atendimento aos pacientes e de prevenção de possível exposição a riscos biológicos, é necessário haver discussão entre os funcionários dos procedimentos executados e informação e programas de prevenção de riscos ocupacionais.

Tendo em vista a importância do trabalho desenvolvido por este serviço e ao risco ocupacional relacionado ao contato com sangue e fluidos corporais durante o atendimento emergencial, sugere-se que temas relacionados ao controle de disseminação de microrganismos sejam discutidos e analisados pela equipe durante os programas de educação continuada, visando melhorar o nível de conhecimento sobre precauções padrão entre profissionais da equipe de atendimento de emergência; possibilitando assim um fórum de discussão entre os funcionários sobre os procedimentos executados e informações que os sensibilizem a promover programas de prevenção de riscos ocupacionais.

\section{REFERÊNCIAS}

1. Brasil. Ministério da Saúde. Portaria n. 2048 de 5 de setembro de 2002. Dispõe sobre a organização do atendimento móvel de urgência - SAMU. Diário Oficial da União, Brasília (DF) 2002; Set 6.

2. Barrios JP, Pérez IO, Cábal FC. Sistema médico prehospitalario de emergencia especializado en medicina mrítica, a 10 años de operación en la Ciudad de México. Neumología y Cirugía de Tórax. 2000;59(4):102-8.
3. Siegel JD, Rhinehart E, Jackson M, Chiarello Linda. Guideline for isolation precautions: preventing transmission of infectious agents in healthcare settings. [cited 2006 Sept 15]. 2007. 219 p. Available from: < http://www.cdc.gov/ ncidod/dhqp/pdf/guidelines/ isolation2007.pdf > .

4. Garner JS, Hospital Infection Control Practices Advisory Commitee. Guideline for isolation precautions in hospitals. Infect Control Hosp Epidemiol. 1996;17(2):53-80. 
5. Phipps W, Honghong W, Min Y, Burgess J, Pellico L, Watkins CW, Guoping H, Williams A. Risk of medical sharps injuries among chinese nurses. Infect Control Hosp Epidemiol. 2006; 34(4): 277-82.

6. Zapparoli AS, Marziale MHP. Risco ocupacional em unidades de suporte básico e avançado de vida em emergências. Rev Bras Enferm. 2006;59(1):41-6.

7. Kermode M, Jolley D, Langkham B, Thomas MS, Holmes W, Gifford SM. Compliance with universal/standard precautions among health aare workers in rural north India. Am J Infect Control. 2005;33(1):27-33.

8. Sax $H$, Perneger $T$, Hugonnet $S$, Herrault $P$, Chraiti $M N$, Pittet D. Knowledge of standard and precautions in a large teaching hospital. Infect Control Hosp Epidemiol. 2005;26(3):298-304.

9. Lopes, ACS; Oliveira, AC; Silva, JT; Paiva, MHRS. Adesão às precauções padrão pela equipe do atendimento pré-hospitalar móvel de Belo Horizonte, Minas Gerais, Brasil. Cad. Saúde Pública. 2008;24(6):1387-1396.

10. Florêncio VB, Rodrigues CA, Pereira MS, Souza ACS. Adesão as precauções padrão entre os profissionais da equipe de resgate pré-hospitalar do corpo de bombeiros de Goiás. Rev. Eletr. Enf. [Internet] 2003;5(1):43-8.

11. Montoya AOD. Piaget: imagem mental e construção do conhecimento. São Paulo: UNESP; 2005. Nishide Vm, Benatti MCC. Riscos ocupacionais entre trabalhadores de enfermagem de uma unidade de terapia intensiva. Rev Esc Enferm USP. 2004;38(4):406-14.
12. Garcia LP, Blank VLG. Condutas pós-exposição ocupacional a material biológico na odontologia. Rev. Saúde Públ. 2008; 42(2): 279-286.

13. Cooper S, O'Carroll J, Jenkin A, Badger B. Collaborative practices in unscheduled emergency care: role and impact of the emergency care practitioner-qualitative and summative findings. EMJ Online. 2007;24(9):625-9.

14. Nishide VM, Benatti MCC. Riscos ocupacionais entre trabalhadores de enfermagem de uma unidade de terapia intensiva. Rev Esc Enferm USP. 2004;38(4):406-14.

15. Sêcco IAO, Leroux AMR, Santos CF, Robazzi MLCC. Epidemiologia dos acidentes de trabalho com material biológico na equipe de enfermagem de um hospital público do Paraná. UNOPAR Cient. Ciênc. Biol. Saúde. 2002;4(1):37-43.

16. Mathews R, Leiss JK, Lyden JT, Sousa S, Ratcliffe JM, Jagger J. Provision and use of personal protective equipment and safety devices in the national study to prevent blood exposure in paramedics. Am j infect control. 2008;36(10):743-749.

17. Angtuaco TL, Oprescu FG, Lal SK, Pennington JH, Russell BD, Co JM, Howden CW.. Universal precautions guidelines: self-reported compliance by gastroenterologists and gastrointestinal endoscopy nurses - a decade's lack of progress. Am J Gastroenterol. 2003;98(11):2420-23.

18. Cutter J, Jordan S. Uptake of guidelines to avoid and report exposure to blood and body fluids. J Adv Nurs. 2004;46(4):441-52. 